\title{
PAPER
}

\section{Transoesophageal echocardiography in selecting patients for anticoagulation after ischaemic stroke or transient ischaemic attack}

\author{
M Strandberg, R J Marttila, H Helenius, J Hartiala
}

See end of article for authors' affiliations

....................

Correspondence to: Dr M Strandberg, Turku University Hospital,

Department of Clinical

Physiology,

Kiinamylluynkatu 4-8,

20520 Turku, Finland:

marjatta.strandberg@phks.fi

or

marjatta.strandberg@tyks.fi

Received

23 November 2001

In final revised form

8 February 2002

Accepted 3 April 2002

\begin{abstract}
Objectives: To investigate prospectively the role of transoesophageal echocardiography (TEE) in selecting patients for anticoagulation in an unselected stroke population.

Methods: Transthoracic echocardiography (TTE) and TEE were done in all clinically suitable hospitalised patients $(n=457)$ with transient ischaemic attack or ischaemic stroke in the acute phase during a two year period in Turku University Hospital. 441 patients were successfully evaluated for cardiac sources of embolism using TEE within 31 days of the event.

Results: A major risk factor for a cardiac source of embolism excluding atrial fibrillation, acute myocardial infarction, and prosthetic valve was detected in 10\% of patients and a minor risk factor for a cardiac source of embolism in $46 \%$. When a major risk factor of a cardiac source of embolism was detected using TTE or TEE and no contraindications were present, the patient was given anticoagulation drugs. If a minor risk factor for a cardiac source of embolism was detected, anticoagulation treatment was started after clinical assessment, if no contraindications were present. In $62(14 \%)$ cases, the patient was given oral anticoagulation drugs or the necessity of ongoing anticoagulation treatment was confirmed on the basis of TEE. When these anticoagulation treated patients were evaluated using logistic regression analysis, they were found to have significantly more atrial fibrillation and histories of myocardial infarctions. Moreover, the patients were mainly men. When patients in sinus rhythm and without any history of cardiac disease were analysed, $8 \%$ of patients were found to have been given anticoagulation drugs on the basis of TEE data.

Conclusion: This study suggests that TEE should be used in patients with stroke even without any clinical evidence of cardiac disease when the patients are candidates for anticoagulation.
\end{abstract}

$\mathrm{T}$ ransient ischaemic attacks (TIA) or ischaemic strokes are caused by cardiac embolism in $15-40 \%$ of cases. ${ }^{1-8}$ Transoesophageal echocardiography (TEE) has proved more sensitive than transthoracic echocardiography (TTE) in diagnosing intracardiac sources of embolism. ${ }^{9-16}$ With the use of TEE, a potential cardiac source of embolism has been found in $41-57 \%$ of patients with ischaemic stroke or TIA. ${ }^{92}$

Patients with cardiac disorders diagnosed using TEE may benefit from anticoagulation drug treatment for secondary prevention of recurrent cerebral embolism. ${ }^{3}$ However, the place of echocardiography in selecting patients for anticoagulation remains to be determined. When 19 studies $(\mathrm{n}=3524)$ on the effect of TTE in patients with suspected systemic arterial embolism were evaluated, a new therapeutic concept emerged from TTE in $4 \%$ of the examinations. ${ }^{17}$ Although TEE has improved the visualisation of the left atrium, the mitral and aortic valves, and the aorta, only a few authors have studied the effect of TEE on the decision to use anticoagulation for stroke and TIA. ${ }^{12} 1819$ In one study, ${ }^{12}$ TEE changed management in only $5 \%$ of cases, but the authors did not discuss cases where TEE was used to confirm the necessity of ongoing anticoagulation. In another study, ${ }^{18}$ TEE had influenced the management of $10 \%$ of patients. That study included patients with atrial fibrillation; anticoagulation may thus have been indicated regardless of TEE results. In a third study, ${ }^{19}$ all patients were in sinus rhythm, had non-diagnostic TTE, and had no carotid artery stenosis. TEE changed the management of $10 \%$ of patients. It is noteworthy that study groups in all these previous studies were small, ranging from 30 to 63 patients. As the effect of routine TEE on the decision to start anticoagulation treatment in unselected stroke populations is controversial, we decided to readdress the question in a larger patient population. Moreover, we wanted to determine what kind of patients benefit most from this examination.

\section{PATIENTS AND METHODS}

During a two year period from 1 January 1997 through 31 December 1998, 457 consecutive patients with TIA or ischaemic stroke in the acute phase were routinely admitted to undergo a TEE examination in the Department of Clinical Physiology at Turku University Hospital.

Only 16 of the patients were excluded from the study because of severe swallowing problems on TEE. The remaining 441 patients were successfully evaluated for cardiac sources of embolism using TEE, most of them (85\%) within a week and all within 31 days (mean 5.5 days, median 4.6 days) of the event. The patients' medical records including laboratory and imaging studies were reviewed retrospectively. The findings from TEE, the patients' medical records, and the results of other examinations were collected for statistical analysis.

The basic study population $(\mathrm{n}=441)$ was unselected, and the age of the patients ranged from 21 to 86 years (mean 63, median 65). In this population, 259 (59\%) were men and 182 (41\%) were women. The Ethics Committee of the University of Turku and University Central Hospital of Turku approved the study.

Abbreviations: $\mathrm{Cl}$, confidence interval; $\mathrm{OR}$, odds ratio; TEE, transoesophageal echocardiography; TIA, transient ischaemic attack; TTE, transthoracic echocardiography 
Table 1 Modification of Hart's classification of echocardiographic findings

\begin{tabular}{ll}
\hline Major risk factors & Minor risk factors \\
\hline Left atrial cavity thrombus & Mitral valve prolapse \\
Left atrial appendage thrombus & Mitral annular calcification \\
Left ventricular thrombus & Calcified aortic stenosis \\
Aortic thrombus & Patent foramen ovale \\
Atrial myxoma & Slow flow (spontaneous echo contrast) \\
Vegetation & Atrial septal aneurysm \\
Infective endocarditis & Left ventricular aneurysm \\
Marantic endocarditis & Aortic aneurysm \\
Dilated cardiomyopathy & False tendon \\
Mitral valve stenosis & Aortic arch plaques \\
\hline
\end{tabular}

Abnormalities on transoesophageal echocardiography (TEE) that are potential cardiac sources of embolism are listed as major or minor risk factors. Atrial fibrillation, acute myocardial infarction, and prosthetic valve are also major risk factors but not listed here because they can be identified without TEE.

The echocardiographic examinations were done using the Acuson 128 XP (Acuson Corp, Mountain View, California, USA) echocardiographic system and a 2.5-3.5 MHz scanning frequency linear array transducer for TTE, as well as a $5.0 \mathrm{MHz}$ biplane and a $5.0 \mathrm{MHz}$ multiplane transducer for TEE. TTE was done before TEE in all patients. The TEE study was done in awake patients using lidocaine gel for local pharyngeal anaesthesia; no other medication was needed. Standard imaging TTE and TEE planes (basal short axis, two chamber, four chamber, transgastric short axis and thoracic aorta) were viewed in all patients. ${ }^{20}$ Dimensions were measured from the TTE parasternal long axis view, and an injection of intravenous sterile isotonic saline and Valsalva manoeuvre were routinely given to every patient. The echocardiograms were stored on videotape.

The echocardiographic findings were categorised according to a modification of Hart's classification. ${ }^{21-23}$ Consequently, echocardiographic abnormalities were considered major or minor risk factors for cardiac sources of embolism (table 1). When a major risk factor for a cardiac source of embolus was detected in TEE and no contraindications were present, the patient was given anticoagulation drugs. Atrial fibrillation, acute myocardial infarction, and prosthetic valve were not included in the major risk factors in this study because they are identified before TEE. If a minor risk factor for a cardiac source of embolism was detected, anticoagulation treatment was started after clinical consideration of the probability of cardiac embolism and treatment hazards.

\section{Statistical analysis}

The statistical analyses were done using SAS statistical software (version 8.00 for Windows, SAS Institute, Cary,
North Carolina, USA). The results are expressed as means (SD) and percentages where appropriate. Two by two tables were constructed to analyse associations between the groups. Significance was tested using the $\chi^{2}$ test and Fisher's exact test. The level of significance for testing all null hypotheses was a two tailed $\mathrm{p}<0.05$. Odds ratios (ORs) and confidence intervals (95\% CIs) were calculated for the risk factors. A logistic regression analysis was used to model the profile of the person to be in that group where TEE findings changed or confirmed the necessity of management. The two sample $t$ test was used to compare the mean age of patients receiving anticoagulation drugs with the mean age of the other examined patients.

\section{RESULTS}

Of all 441 studied patients, $73 \%$ had an ischaemic stroke and $27 \%$ had a TIA. Table 2 shows a more specific division by subtype. Coronary artery disease was present in $24 \%$ of the patients and $50 \%$ of them also had a history of myocardial infarction, and three patients had an acute myocardial infarction when hospitalised. Of these patients, $23 \%$ had undergone coronary artery bypass or percutaneous transluminal coronary angioplasty. Of all examined patients, $7 \%$ had had manifestations of congestive heart failure, $2 \%$ had a history of valve disease, and only $2 \%$ had been given anticoagulation drugs previously. Fourteen per cent of the patients had a history of chronic $(2 \%)$ or paroxysmal ( $12 \%)$ atrial fibrillation.

We identified a major or a minor risk factor for a cardiac source of embolism (table 1) in $49 \%$ of patients examined, and when the plaques in the descending aorta were included, in $57 \%$. A major risk factor was identified in $10 \%$ of patients and a minor risk factor in $46 \%$ in the study population. Of those with stroke or TIA, 62 (14\%) were being given anticoagulation drugs or their ongoing anticoagulation treatment was justified on the basis of TEE. In 17 of the 62 patients, the suspected source of embolism had already been detected on TTE, but even in these cases TEE often gave useful additional information (eight thrombi, two slow flows, and four aortic plaques). Only in five cases did TEE not gave additional information.

Patients with atrial fibrillation had significantly more major $(\mathrm{p}<0.001$, OR 6.8, 95\% CI 3.5 to 13.3) and minor ( $\mathrm{p}=0.01$, OR $2.1,95 \%$ CI 1.2 to 3.6 ) risk factors for cardiac sources of embolism than those in sinus rhythm. If patients with atrial fibrillation were excluded, 36 of 381 (9\%) were still receiving anticoagulation drugs on the basis of TEE data: 23 of 36 because of a major and 13 of 36 because of a minor risk factor for a cardiac source.

In the anticoagulation treated group $(\mathrm{n}=62), 41$ thrombi were found on TEE (table 3 ). Of these, 21 were in the left atrial appendage, eight in the descending aorta, seven in the aortic

$\begin{aligned} & \text { Table } 2 \text { Classification of ischaemic stroke and transient ischaemic strokes (TIA) by } \\
& \text { subtypes }\end{aligned}$
\begin{tabular}{llll} 
& & \\
\hline & No (\%) & Male (\%) & Mean (SD) age (years) \\
\hline Transient ischemic attack & $68(16)$ & 59 & $59(11)$ \\
$\quad$ Carotid TIA & $50(11)$ & 64 & $64(9)$ \\
Vertebrobasilar TIA & $9(2)$ & 56 & $65(3)$ \\
Infarction & $186(42)$ & 58 & $64(11)$ \\
Anterior cerebral artery & $32(7)$ & 53 & $61(12)$ \\
Middle cerebral artery & $51(12)$ & 59 & $63(11)$ \\
Posterior cerebral artery & $24(5)$ & 63 & $59(12)$ \\
Brainstem & $21(5)$ & 57 & $65(8)$ \\
Cerebellum & $441(100)$ & & \\
Lacunar infarction &
\end{tabular}
Total

The patients were divided into subgroups by a neurologist on the basis of computed tomography or magnetic resonance imaging findings, or of clinical manifestations. 
Table 3 Cardiac risk factors for embolism detected using transthoracic echocardiography (TTE) and TEE in 441 unselected patients with stroke or TIA

\begin{tabular}{|c|c|c|}
\hline Cardiac risk factors for embolism & $\begin{array}{l}\text { Detected by TTE } \\
\text { No (\%) }\end{array}$ & $\begin{array}{l}\text { Detected by TEE } \\
\text { No }(\%)\end{array}$ \\
\hline \multicolumn{3}{|l|}{ Major risk factors } \\
\hline Left atrial cavity thrombus & $2(0.5)$ & * \\
\hline Left atrial appendage thrombus & 0 & $21(4.8)$ \\
\hline Left ventricular thrombus & $2(0.5)$ & * \\
\hline Aortic thrombus & 0 & $16(3.6)$ \\
\hline Dilated cardiomyopathy with ejection fraction $<35 \%$ & $7(1.6)$ & * \\
\hline Mitral valve stenosis & $1(0.2)$ & * \\
\hline \multicolumn{3}{|l|}{ Minor risk factors } \\
\hline Mitral valve prolapse & $21(4.8)$ & * \\
\hline Mitral annular calcification & $8(1.8)$ & * \\
\hline Calcified aortic stenosis & $6(1.4)$ & * \\
\hline Patent foramen ovale & 0 & $67(15.2)$ \\
\hline Slow flow (spontaneous echo contrast) & 0 & $20(4.5)$ \\
\hline Atrial septal aneurysm & $20(4.5)$ & * \\
\hline Left ventricular aneurysm & $10(2.3)$ & * \\
\hline Aortic aneurysm & 0 & $1(0.2)$ \\
\hline False tendon & $7(1.6)$ & * \\
\hline Aortic plaques & 0 & $73(16.6)$ \\
\hline
\end{tabular}

arch, one in the left ventricle, one in a left ventricular aneurysm, one in an aortic arch aneurysm, one in an atrial septal aneurysm, and one attached to a prosthetic mitral valve. Of the patients receiving anticoagulation drugs, 15 of $62(24 \%)$ had a patent foramen ovale. Twelve of these 15 patients were receiving anticoagulation drugs on the basis of the patent foramen ovale, and in all these cases pronounced right to left shunting was seen on Valsalva manoeuvre. In 12 of 62 (19\%) cases, the left ventricle was dilated, but in only two was it in the patient's recorded history. Slow flow (spontaneous echo contrast) was detected in 16 of $62(26 \%)$ patients, and in five anticoagulation treatment was started mainly for this reason. Two of the patients were treated mainly for left ventricular wall motion abnormality and two for left ventricular aneurysm. Thirteen cardiac aneurysms were found in the anticoagulation treated group, and none of these cardiac aneurysms had been identified before the examination.

We analysed more closely the group of 62 patients in whom TEE changed or confirmed the necessity of management. The age of the patients ranged from 21 to 86 years (mean 63, median 65), and the difference in age was not significant $(\mathrm{p}=0.27)$ between them and the rest of the patients $(\mathrm{n}=379)$ examined, here considered to be the nonanticoagulation treated group on the basis of TEE. No significant dominance of any subtype of infarction or TIA was seen. Of patients receiving anticoagulation drugs, 49 of 62 (79\%) were men, significantly $(\mathrm{p}<0.001)$ more than women (table $4)$, and $26(42 \%)$ had atrial fibrillation, which was naturally significantly more $(\mathrm{p}<0.001)$ than the rate for the nonanticoagulation treated group. Significantly more had coronary artery disease $(p=0.006)$. These patients significantly more often had histories of myocardial infarction $(p=0.003)$ than patients in the non-anticoagulation treated group on the basis of TEE. Significantly more had histories of valve disease $(p=0.016)$ and congestive heart failure $(p=0.002)$. Only 7 of $62(11 \%)$ patients received anticoagulation drugs when they experienced their ischaemic attacks. Two of the patients had a prosthetic mitral valve, and in five of seven patients anticoagulation treatment was inadequate.

In this study, 26 of 62 patients could already have been given anticoagulation drugs on the basis of atrial fibrillation. There were still 36 patients in sinus rhythm who were treated on the basis of their TEE findings. When these 36 patients were analysed more closely, significantly more men ( $p<0.001$, OR 5.1, 95\% CI 1.9 to 13.5 ) were seen in this group with a significantly higher prevalence of coronary artery disease $(\mathrm{p}=0.02$, OR $2.4,95 \%$ CI 1.2 to 5.0$)$ and previous myocardial infarctions $(p=0.02$, OR $3.0,95 \%$ CI 1.3 to 6.8$)$ than were seen in other patients in sinus rhythm.

When the patients in sinus rhythm and with no history of cardiac disease were evaluated as an independent group, in 22 of $286(8 \%)$ patients anticoagulation treatment appeared to be based on TEE data. In this group, a major risk factor for a cardiac source of embolism was found in 5\% of patients and a minor risk factor for a cardiac source of embolism in 39\%.

On logistic regression analysis $(n=441)$, adjusted by the other variables, the strongest predictor of the effect of TEE on

Table 4 Cerebrovascular risk factors and their prevalences in patients who received anticoagulation drugs on the basis of TEE findings and the other patients examined

\begin{tabular}{|c|c|c|c|c|c|}
\hline & $\begin{array}{l}\text { Anticoagulation } \\
(n=62) \text { No }(\%)\end{array}$ & $\begin{array}{l}\text { No anticoagulation } \\
(n=379) \text { No }(\%)\end{array}$ & $p$ Value & OR & $95 \% \mathrm{Cl}$ \\
\hline Men & 49 (79) & $210(55)$ & $<0.001$ & 3.0 & 1.6 to 5.8 \\
\hline Atrial fibrillation & $26(42)$ & $34(9.0)$ & $<0.001$ & 7.3 & 4.0 to 13.6 \\
\hline Coronary artery disease & $24(39)$ & $81(21)$ & 0.006 & 2.3 & 1.3 to 4.1 \\
\hline Previous myocardial infarction & $15(24)$ & $37(9.8)$ & 0.003 & 3.0 & 1.5 to 5.8 \\
\hline Valve disease & $4(6.5)$ & $4(1.0)$ & 0.016 & 6.5 & 1.6 to 26.6 \\
\hline Congestive heart failure & $11(18)$ & $21(5.5)$ & 0.002 & 3.7 & 1.7 to 8.1 \\
\hline Hypertension (medication) & 20 (33) & 148 (39) & NS & 0.7 & 0.4 to 1.3 \\
\hline Hypercholesterolaemia (medication) & $8(13)$ & $28(7.4)$ & NS & 1.9 & 0.8 to 4.3 \\
\hline Diabetes & $16(26)$ & 84 (22) & NS & 1.2 & 0.7 to 2.3 \\
\hline
\end{tabular}

$\mathrm{Cl}$, confidence interval; $\mathrm{OR}$, odds ratio. 
the decision to give the patient anticoagulation drugs was, as expected, atrial fibrillation ( $p<0.001$, OR $7.7,95 \%$ CI 4.0 to 14.7). The other significant predictors were male sex ( $\mathrm{p}<0.003$, OR $2.8,95 \% \mathrm{CI} 1.4$ to 5.6 ) and previous myocardial infarction ( $\mathrm{p}=0.005$, OR $2.9,95 \%$ CI 1.4 to 6.1$)$.

\section{DISCUSSION}

TEE is a useful tool for detecting potential intracardiac or aortic sources of embolism. It has proved to be superior to TTE in the identification of structural changes related to possible cardiac embolism, especially in the absence of any clinical evidence of cardiac disease. ${ }^{11}{ }^{23}$ Such cardiac changes include thrombi, patent foramen ovale, atrial septal aneurysm, slow left atrial flow, and myxomatous degeneration of the mitral valve associated with mitral valve prolapse. ${ }^{10}$ However, it remains to be determined whether all patients should undergo TEE. Previous studies with TEE have shown a high frequency of cardiac changes in patients with stroke, not only in those with but also in those without heart disease. ${ }^{10} 11132425$ No established guidelines for patient selection are available yet.

Our study was done to determine the usefulness of TEE as a routine examination in unselected patients with stroke. TEE was done in 441 patients with ischaemic stroke or TIA very soon after the onset of symptoms (mean 5.5 days), and in 62 (14\%) cases it led to anticoagulation treatment. In some cases, TEE was done rather late and something important may therefore have been overlooked. When all patients with atrial fibrillation were excluded from the analysis, it was found that TEE caused a change in or confirmed the appropriateness of management of 36 of 381 (9\%) patients.

The presence of a major risk factor for a cardiac source of embolism (table 1) or some of the minor risk factors detected by TEE may predict future thromboembolic events, indicating the need for long term anticoagulation treatment. Cardiac evaluation may be indicated in patients even when a coexistent explanation for the event exists, for example, when a cerebral ischaemic event can be clinically associated with atherosclerotic disease of the carotid artery. Reportedly, 13 to $48 \%$ of patients with a potential cardiac source of embolism have significant cerebrovascular atherosclerosis. ${ }^{3}$ For example, when patients with critical carotid stenosis on carotid ultrasound in our study were analysed as a group, 8 of 47 (17\%) were found to have been given anticoagulation drugs on the basis of TEE findings, although a coexistent explanation was also available.

Some clinical factors have been independently associated with stroke in previous analyses. These include increasing age, previous TIA or stroke, history of diabetes, history of heart failure, or history of hypertension. ${ }^{26}{ }^{27}$ We found in our analysis that patients with an identified cardiac source of embolism were significantly more often men and more often had coronary artery disease, previous myocardial infarction, known valve disease, myocardial insufficiency, or atrial fibrillation.

The decision to do TEE after stroke or TIA should be based on the likelihood of the findings contributing to the patient's management. Consequently, TEE should not be considered if the patient is not a candidate for anticoagulation treatment. An algorithm has already been proposed for the use of TEE in evaluating patients with stroke or TIA ${ }^{28}$ Considering that most patients with atrial fibrillation are candidates for empirical anticoagulation treatment, the use of this algorithm would limit the use of TEE to patients with clinical evidence of heart disease but without atrial fibrillation and to those with TTE findings suggestive of a severe abnormality. This would decrease the number of TEEs by at least two thirds. However, in our study, where TEE was done in an unselected stroke population, there were reasonably many patients in sinus rhythm with no history of cardiac disease with an obvious cardiac source of embolism (22 of 286). Of these patients, 5\% had a major risk factor for a cardiac source of embolism and
$39 \%$ had a minor risk factor and, consequently, $8 \%$ of these patients were given anticoagulation drugs solely on the basis of TEE findings. In conclusion, the effect of TEE on the decision to give anticoagulation drugs was found to be higher than previously reported. Therefore, TEE should be used more often in patients with stroke or TIA who are candidates for receiving anticoagulation drugs and in sinus rhythm.

\section{ACKNOWLEDGEMENTS}

We thank Päivi Sandberg, Tuula Raitanen, and Simo Merne for help.

\section{Authors' affiliations}

M Strandberg, J Hartiala, Department of Clinical Physiology, Turku

University Hospital, Turku, Finland

R J Marttila, Department of Neurology, Turku University Hospital, Turku, Finland

H Helenius, Department of Biostatistics, University of Turku, Turku,

Finland.

Competing interests: none declared

\section{REFERENCES}

1 Sorensen PS, Boysen G, Jensen G, et al. Prevalence of stroke in a district of Copenhagen. The Copenhagen city heart study. Acta Neurol Scand 1982:66:68-81

2 Norrving B, Löwenheim P. Epidemiology of stroke in Lund-Orup, Sweden, 1983-85. Incidence of first stroke and age-related changes in subtypes. Acta Neurol Scand 1988;78:408-13.

3 Cerebral Embolism Task Force. Cardiogenic brain embolism: the second report of the Cerebral Embolism Task Force. Arch Neurol 1989;46:727-43.

4 Olsen TS, Skriver EB, Herning M. Cause of cerebral infarction in the carotid territory: its relation to the size and location of the infarct and to the underlying vascular lesion. Stroke 1985;16:459-66.

5 Bogousslavsky J, Melle GV, Regli F. The Lausanne stroke study: analysis of 1000 consecutive patients with first stroke. Stroke 1988;19:1083-92.

6 Bogousslavsky J, Hachinski VC, Boughner DR, et al. Cardiac and arterial lesions in carotid transient ischemic attacks. Arch Neurol 1986:43:223-8.

7 Sacco RL, Foulkes MA, Mohr JP, et al. Determinants of early recurrence of cerebral infarction: the stroke data bank. Stroke 1989;20:983-9.

8 Comess KA, DeRook FA, Beach KW, et al. Transesophageal echocardiography and carotid ultrasound in patients with cerebral ischemia: prevalence of findings and recurrent stroke risk. J Am Coll Cardiol 1994;23:1598-603.

9 Pearson AC, Labovitz AJ, Tatineni S, et al. Superiority of transesophageal echocardiography in detecting cardiac source of embolism in patients with cerebral ischemia of uncertain etiology. J Am Coll Cardiol 1991:17:66-72.

10 Lee RJ, Batzokis T, Yeoh TK, et al. Enhanced detection of intracardiac sources of cerebral emboli by transesophageal echocardiography. Stroke 1991;22:734-9.

11 Aschenberg W, Schlueter $M$, Kremer $P$, et al. Transesophageal two-dimensional echocardiography for detection of left atrial appendage thrombus. J Am Coll Cardiol 1986;7:163-6.

12 Cujec B, Polasek P, Voll C, et al. Transesophageal echocardiography in detection of potential cardiac source of embolism in stroke patients. Stroke 1991;22:727-33.

13 Pop G, Sutherland GR, Koudstaal PJ, et al. Transesophageal echocardiography in the detection of intracardiac embolic sources in patients with transient ischemic attacks. Stroke 1990;21:560-5

14 Mugge A, Daniel WG, Grank G, et al. Echocardiography in infectious endocarditis: reassessment of prognostic implications of vegetation size determined by the transthoracic and the transesophageal approach. J Am Coll Cardiol 1989;14:631-6.

15 Hofmann T, Kasper W, Meinertz T, et al. Echocardiographic evaluation of patients with clinically suspected arterial emboli. Lancet 1990;336:1421-4.

16 Tunick PA, Perez JL, Kronzon I. Protruding atheromas in the thoracic aorta and systemic embolization. Ann Intern Med 1991;1 15:423-7.

17 Egeblad H, Andersen K, Hartiala J, et al. Role of echocardiography in systemic arterial embolism: a review with recommendations. Scand Cardiovasc J 1998;32:323-42.

18 Hata JS, Ayres RW, Biller J, et al. Impact of transesophageal echocardiography on the anticoagulation management of patients admitted with focal cerebral ischemia. Am J Cardiol 1993;72:707-10.

19 Gunter R, Michael F, Florentin A. Transesophageal echocardiography in patients with focal cerebral ischemia of unknown cause. Stroke 1996;27:691-4.

20 Seward JB, Khandheria BK, Oh JK, et al. Transesophageal echocardiography: technique, anatomic correlations, implementation, and clinical applications. Mayo Clin Proc 1988;63:649-80.

21 Hart RG. Cardiogenic embolism to the brain. Lancet 1992;329:58994. 
22 Roijer A, Lindgren A, Algotsson L, et al. Cardiac changes in stroke patients and controls evaluated with transesophageal echocardiography. Scand Cardiovasc J 1997;31:329-37.

23 Hausmann D, Mugge A, Daniel WG. Identification of patent foramen ovale permitting paradoxical embolism. J Am Coll Cardiol 1995:26:1030-8.

24 Black IW, Hopkins AP, Jacobsson BM, et al. Role of transesophageal echocardiography in evaluation of cardiogenic embolism. Br Heart J 1991;66:302-7.
25 Zenker G, Erbel R, Krämer G, et al. Transesophageal two-dimensional echocardiography in young patients with cerebral ischemic events. Stroke 1988; 19:345-8

26 AFI Investigators. Risk factors for stroke and efficacy of antithrombotic therapy in atrial fibrillation. Arch Intern Med 1994;154:1449-57.

27 AFI Investigators. Echocardiographic predictors of stroke in patients with atrial fibrillation. Arch Intern Med 1998;158:1316-20.

28 Warner MF, Momah KI. Routine transesophageal echocardiography for cerebral ischemia. Is it really necessary? Arch Intern Med 1996;156:1719-23.

\section{Call for peer reviewers}

Clinical Evidence is a regularly updated evidence based journal available world wide both as a paper version and on the internet. Clinical Evidence urgently needs to recruit a number of new contributors. Contributors are health care professionals or epidemiologists with experience in evidence based medicine and the ability to write in a concise and structured way.

Clinical Evidence needs to recruit a number of new peer reviewers. Peer reviewers are health care professionals or epidemiologists with experience in evidence based medicine. As a peer reviewer you would be asked for your views on the clinical relevance, validity and accessibility of specific topics within the journal, and their usefulness to the intended audience (international generalists and health care professionals, possibly with limited statistical knowledge). Topics are usually 2000-3000 words in length and we would ask you to review between 2-5 topics per year. The peer review process takes place throughout the year, and our turnaround time for each review is ideally 10-14 days.

If you are interested in becoming a peer reviewer for Clinical Evidence, please complete the peer review questionnaire at www.clinicalevidence.com or contact Polly Brown (pbrown@bmigroup.com). 